\title{
Projections Onto Convex Sets (POCS) Based Optimization by Lifting
}

\author{
A. Enis Cetin**, A. Bozkurt, O. Gunay, Y. H. Habiboglu, K. Kose*, I. Onaran, M. Tofighi, R. A. Sevimli \\ Department. of Electrical and Electronic Engineering, Bilkent University, Ankara, Turkey \\ ${ }^{*}$ Dermatology Service, Memorial Sloan Kettering Cancer Center, New York, New York \\ ${ }^{* *}$ cetin at bilkent.edu.tr
}

A new optimization technique based on the projections onto convex space (POCS) framework for solving convex and some nonconvex optimization problems are presented. The dimension of the minimization problem is lifted by one and sets corresponding to the cost function are defined. If the cost function is a convex function in $R^{N}$ the corresponding set which is the epigraph of the cost function is also a convex set in $R^{N+1}$. The iterative optimization approach starts with an arbitrary initial estimate in $R^{N+1}$ and an orthogonal projection is performed onto one of the sets in a sequential manner at each step of the optimization problem. The method provides globally optimal solutions in total-variation, filtered variation, $l_{1}$, and entropic cost functions. It is also experimentally observed that cost functions based on $l_{p}, p<1$ may be handled by using the supporting hyperplane concept. The new POCS based method can be used in image deblurring, restoration and compressive sensing problems.

In many inverse signal and image processing problems and compressing sensing problems an optimization problem is solved to find a solution:

$$
\min _{\mathbf{w} \in \mathrm{C}} f(\mathbf{w})
$$

where $C$ is a set in $\mathbb{R}^{N}$ and $f(\mathbf{w})$ is the cost function. Bregman developed iterative methods based on the so-called Bregman distance to solve convex optimization problems. In Bregman's approach, it is necessary to perform a D-projection (or Bregman projection) onto a convex set at each step of the algorithm [1], [2]. Unfortunately it may not be easy to compute the Bregman projections in general.

In this article, Bregman's projections onto convex sets (POCS) framework is used to solve convex and some non-convex optimization problems without using the Bregman distance approach.

We increase the dimension by one and define the following sets in $\mathbb{R}^{N+1}$ corresponding to the cost function $f(\mathbf{w})$ as follows:

$$
\mathrm{C}_{f}=\left\{\underline{\mathbf{w}}=\left[\begin{array}{ll}
\mathbf{w}^{T} & y
\end{array}\right]^{T}: y \geq f(\mathbf{w})\right\}
$$

which is the set of $N+1$ dimensional vectors whose $N+1^{\text {st }}$ component $y$ is greater than $f(\mathbf{w})$. The second set that is related with the cost function $f(\mathbf{w})$ is the level set:

$$
\mathrm{C}_{s}=\left\{\underline{\mathbf{w}}=\left[\mathbf{w}^{T} y\right]^{T}: y \leq \alpha, \underline{\mathbf{w}} \in \mathbb{R}^{N+1}\right\}
$$

where $\alpha$ is a real number. Here it is assumed that $f(\mathbf{w}) \geq \alpha$ for all $f(\mathbf{w}) \in \mathbb{R}$ such that the sets $\mathrm{C}_{\mathrm{f}}$ and $\mathrm{C}_{\mathrm{s}}$ do not intersect. They are both closed and convex sets in $\mathbb{R}^{N+1}$. Sets $\mathrm{C}_{\mathrm{f}}$ and $\mathrm{C}_{\mathrm{s}}$ are graphically illustrated in Fig. 1 in which $\alpha=0$. The POCS based minimization algorithm starts with an arbitrary $\underline{\mathbf{w}}_{0}=\left[\begin{array}{ll}\mathbf{w}_{0}^{T} & y_{0}\end{array}\right]^{T} \in \mathbb{R}^{N+1}$. We project $\underline{\mathbf{w}}_{0}$ onto the set $\mathrm{C}_{\mathrm{s}}$ to obtain the first iterate $\underline{\mathbf{w}}_{1}$ which will be,

$$
\underline{\mathbf{w}}_{1}=\left[\begin{array}{ll}
\mathbf{w}_{0}^{T} & 0
\end{array}\right]^{T}
$$

where $\alpha=0$ is assumed as in Fig. 1 . Then we project $\underline{\mathbf{w}}_{1}$ onto the set $\mathrm{C}_{\mathrm{f}}$. The new iterate $\underline{\mathbf{w}}_{2}$ is determined by minimizing the distance

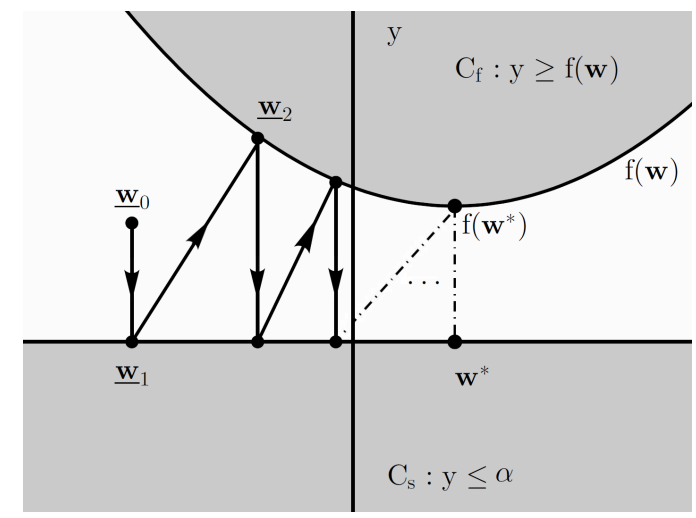

Fig. 1. Two convex sets $\mathrm{C}_{\mathrm{f}}$ and $\mathrm{C}_{\mathrm{S}}$ corresponding to the cost function $f$. We sequentially project an initial vector $\underline{\mathbf{w}}_{0}$ onto $C_{s}$ and $C_{f}$ to find the global minimum which is located at $\mathrm{w}^{*}$.

between $\underline{\mathbf{w}}_{1}$ and $\mathrm{C}_{\mathrm{f}}$, i.e.,

$$
\underline{\mathbf{w}}_{2}=\arg \min _{\underline{\mathbf{w}} \in \mathrm{C}_{\mathrm{s}}}\left\|\underline{\mathbf{w}}_{1}-\underline{\mathbf{w}}\right\|
$$

After finding $\underline{\mathbf{w}}_{2}$, we perform the next projection onto the set $\mathrm{C}_{\mathrm{s}}$ and obtain $\underline{\mathbf{w}}_{3}$ etc. Eventually iterates oscillate between two nearest vectors of the two sets $\mathrm{C}_{\mathrm{s}}$ and $\mathrm{C}_{\mathrm{f}}$. As a result we obtain

$$
\lim _{n \rightarrow \infty} \underline{\mathbf{w}}_{2 n}=\left[\begin{array}{ll}
\mathbf{w}^{*} & f\left(\mathbf{w}^{*}\right)
\end{array}\right]^{T}
$$

where $\mathbf{w}^{*}$ is the $N$ dimensional vector minimizing $f(\mathbf{w})$. The proof of Equation (6) follows from Bregman's POCS theorem. It was generalized to non-intersection case by Gubin et. al. [3]. A more detailed version of the proof of convergence together with a complete list of references is available in [4].

\section{REFERENCES}

[1] L. Bregman, "The Relaxation Method of Finding the Common Point of Convex Sets and Its Application to the Solution of Problems in Convex Programming," $\{$ USSR $\}$ Computational Mathematics and Mathematical Physics, vol. 7, no. 3, pp. 200 - 217, 1967. [Online]. Available: http://www.sciencedirect.com/science/article/pii/0041555367900407

[2] - "Finding the common point of convex sets by the method of successive projection.(russian)," $\{$ USSR $\}$ Dokl. Akad. Nauk SSSR, vol. 7, no. 3, pp. 200 - 217, 1965. [Online]. Available: http: //www.sciencedirect.com/science/article/pii/0041555367900407

[3] L. Gubin, B. Polyak, and E. Raik, "The Method of Projections for Finding the Common Point of Convex Sets," $\{U S S R\}$ Computational Mathematics and Mathematical Physics, vol. 7, no. 6, pp. 1 - 24, 1967. [Online]. Available: http://www.sciencedirect.com/science/article/ pii/0041555367901139

[4] A. E. Cetin, A. Bozkurt, O. Gunay, H. Y. H., K. K., I. Onaran, and S. R. A., "Projections onto convex sets (pocs) based optimization by lifting,", ArXiv e-prints, vol. arXiv:1306.2516 [math.OC], 2013. 